\title{
Application of an Optimal Preview Control for Simulation of Closed-Loop Automobile Driving
}

\author{
CHARLES C. MACADAM
}

\begin{abstract}
An optimal preview control method is applied to the automobile path following problem. The technique is first used to examine the straight-line regulatory driving task and results compared with similar experimental measurements. The method is further demonstrated by closedloop simulation of an automobile driver/vehicle system during transient lane-change maneuvers. The computer simulation results are compared with equivalent vehicle test measurements.
\end{abstract}

\section{INTRODUCTION}

$\mathrm{T}$ HIS PAPER presents example applications (to the automobile path following problem) of a general method of control synthesis presented in [1]. The method is demonstrated here by simulation of a closed-loop automobile/driver system and the results compared with driver/vehicle test measurements. Results for the optimal preview control are also discussed within the context of manual control pursuit tracking task findings.

The control technique demonstrated herein is designed for application to linear time-invariant systems utilizing preview control strategies for regulation or tracking tasks. A common example of this type of control strategy occurs during normal automobile path following in which drivers "look-ahead" to follow a desired path. Human operators, as part of various man-machine systems, typically employ preview control strategies to control and stabilize such systems. It is widely recognized that human operators are capable of controlling and adapting to a wide variety of dynamical systems, many of which are vehicles with preview-oriented control requirements such as automobiles, bicycles, and complex aircraft [2]-[8]. Clearly human control of most vehicles would not be possible without some training by the operator to acquire an understanding of the vehicle response to various control inputs. While a certain portion of this training serves to identify and reinforce learned open-loop responses for repeated and familiar control task scenarios, the remainder frequently serves to identify and reinforce the operator's understanding or "feel" of the vehicle response to control inputs continually in use for closed-loop regulation and/or pursuit needs. It is in this latter control category for general linear system representations capable of preview control strategies, that the method presented in [1] can find particular application. As will be demonstrated in this paper, application to the

Manuscript received October 10, 1980; revised March 2, 1981.

The author is with the Highway Safety Research Institute of the University of Michigan, Ann Arbor, MI 48109. automobile path following problem produces substantive agreement when compared with driver/vehicle experimental measurements for both straight-line regulatory driving and transient lane-change maneuvers.

\section{The Optimal Preview Control}

Before applying the optimal preview control of [1] to the automobile path following problem, the main results and symbol definitions contained therein are briefly reviewed in this section for later reference. As derived in [1], for the linear system

where

$$
\begin{aligned}
& \dot{\boldsymbol{x}}=F \boldsymbol{x}+g u \\
& y=\boldsymbol{m}^{T} \boldsymbol{x}
\end{aligned}
$$

$\boldsymbol{x} \quad n \times 1$ state vector,

$y$ scalar output related to the state by the $n \times 1 m^{T}$ constant observer vector transpose,

$F$ constant $n \times n$ system matrix, and

g constant $n \times 1$ control coefficient vector,

the optimal control $u^{0}(t)$ which minimizes a special form of the local performance index,

$$
J \triangleq \frac{1}{T} \int_{t}^{t+T}\{[f(\eta)-y(\eta)] W(\eta-t)\}^{2} d \eta
$$

over the current preview interval $(t, t+T)$ where

$W$ arbitrary weighting function over the preview interval

and

$f$ previewed input,

is given by

$$
\begin{aligned}
& u^{0}(t)=\left[\int_{t}^{t+T}\left\{f(\eta)-\boldsymbol{m}^{T}\left[I+\sum_{n=1}^{\infty} \frac{F^{n}(\eta-t)^{n}}{n !}\right] \boldsymbol{x}(t)\right\}\right. \\
& \cdot\left.\left\{(\eta-t) \boldsymbol{m}^{T}\left[I+\sum_{n=1}^{\infty} \frac{F^{n}(\eta-t)^{n}}{(n+1) !}\right] \boldsymbol{g}\right\} W(\eta-t) d \eta\right] \\
& /\left[\int_{t}^{t+T}\left\{(\eta-t) \boldsymbol{m}^{T}\left[I+\sum_{n=1}^{\infty} \frac{F^{n}(\eta-t)^{n}}{(n+1) !}\right] \boldsymbol{g}\right\}^{2}\right. \\
&\cdot W(\eta-t) d \eta]
\end{aligned}
$$


where $I$ is the identity matrix. For the special case of $W(\eta-t)=\delta\left(T^{*}\right)$, the Dirac delta function for $0<T^{*} \leqslant$ $T$, (4) simplifies to

$$
\begin{aligned}
u^{0}(t) & =\frac{f\left(t+T^{*}\right)-\boldsymbol{m}^{T}\left[I+\sum_{n=1}^{\infty} \frac{F^{n}\left(T^{*}\right)^{n}}{n !}\right] \boldsymbol{x}(t)}{T^{*} \boldsymbol{m}^{T}\left[I+\sum_{n=1}^{\infty} \frac{F^{n}\left(T^{*}\right)^{n}}{(n+1) !}\right] \boldsymbol{g}} \\
& =\left[f\left(t+T^{*}\right)-y_{0}\left(t+T^{*}\right)\right] /\left(T^{*} K\right),
\end{aligned}
$$

the single-point preview control version of (4), where

$$
K \triangleq \boldsymbol{m}^{T}\left[I+\sum_{n=1}^{\infty} \frac{F^{n}\left(T^{*}\right)^{n}}{(n+1) !}\right] \boldsymbol{g}
$$

Equation (6) represents a proportional controller with gain inversely related to the preview interval $T^{*}$ and operating on the error between the previewed input $f\left(t+T^{*}\right)$ and $y_{0}\left(t+T^{*}\right)$, that portion of the previewed output deriving from the state vector's current initial condition. Likewise (4) can be interpreted as a proportional controller operating on a similar error averaged and weighted over the preview interval $(t, t+T)$ by the additional terms appearing in (4).

It is also shown in [1] that the optimal solution $u^{0}(t)$ can be expressed in terms of any current nonoptimal $u(t)$ and correspondingly nonzero preview output error $\epsilon(t)$ as

$$
u^{0}(t)=u(t)+\frac{\int_{t}^{t+T} \epsilon(\eta) A(\eta) W(\eta-t) d \eta}{\int_{t}^{t+T} A^{2}(\eta) W(\eta-t) d \eta}
$$

where

$$
\begin{aligned}
A(\eta) & \triangleq(\eta-t) \boldsymbol{m}^{T}\left[I+\sum_{n=1}^{\infty} \frac{F^{n}(\eta-t)^{n}}{(n+1) !}\right] \boldsymbol{g} \\
\epsilon(\eta) & \triangleq f(\eta)-\boldsymbol{m}^{T} \phi(\eta, t) x(t)-u(t) A(\eta) \\
\phi(\eta, t) & \triangleq I+\sum_{n=1}^{\infty} \frac{F^{n}(\eta-t)^{n}}{n !} .
\end{aligned}
$$

For the special case of $W(\eta-t)=\delta\left(T^{*}\right)$, as before, (7) reduces to

$$
u^{0}(t)=u(t)+\frac{\epsilon\left(t+T^{*}\right)}{T^{*} \cdot K}
$$

The formulation expressed by (7) can be useful in describing systems which do not achieve, though closely approximate, the defined optimal system behavior. Such cases may arise from limitations in achieving the precise optimal control due to time lags or dynamic properties inherent in the controller and not accounted for a priori in the optimization. The next two sections adopt this view for the car/driver man--machine system in an attempt to describe and explain actual closed-loop driving behavior.

Finally, it was also shown in [1] that information concerning stability of the closed-loop system utilizing the optimal preview control of (4) or (7) is provided by the characteristic roots of the constant matrix

$$
\left[F-g c^{T}\right]
$$

where

$$
\boldsymbol{c}^{T}=\frac{\boldsymbol{m}^{T} \int_{0}^{T} \phi(\eta, 0)\left\{\eta \boldsymbol{m}^{T}\left[I+\sum_{n=1}^{\infty} \frac{F^{n}(\eta)^{n}}{(n+1) !}\right] \boldsymbol{g}\right\} W(\eta) d \eta}{\int_{0}^{T}\left\{\eta \boldsymbol{m}^{T}\left[I+\sum_{n=1}^{\infty} \frac{F^{n}(\eta)^{n}}{(n+1) !}\right] \boldsymbol{g}\right\}^{2} W(\eta) d \eta}
$$

For the special case of $W(\eta)=\delta\left(T^{*}\right)$, (9) becomes

$$
F-\left\{\boldsymbol{g m}^{T}\left[I+\sum_{n=1}^{\infty} \frac{F^{n}\left(T^{*}\right)^{n}}{n !}\right] /\left(T^{*} \cdot K\right)\right\} .
$$

\section{Application to Manual Control Pursuit TRACKING TASKS AS REPRESENTED BY Straight-Line AUtomobile Driving}

The most well-known and characteristic property exhibited by human operators in tracking tasks is the transport delay deriving from perceptual and neuromuscular mechanisms. By introducing this inherent delay property $a$ posteriori in the optimal preview control formulation, excellent agreement can be demonstrated between typical manual control pursuit tracking task results and the resulting optimal preview controller modified to include the inherent transport delay (heretofore referred to as the "modified" optimal preview control).

For reasons of clarity and notational simplicity, the discussion in this section will make use only of $(8)$, the single-point preview control version of (7). Equation (8) can be represented by the block diagram of Fig. 1, where $\boldsymbol{G}(s)=[I s-F]^{-1} \boldsymbol{g}$ represents the controlled element vector transfer function, and $u(t)$, the current control, is related to the optimal control $u^{0}(t)$ by a transfer function $H(s)$ (previously assumed equal to one in the derivation of the optimal control $\left.u^{0}(t)\right)$. The introduction of the $H(s)$ transfer function is useful in describing systems which function (or are presumed to do so) in an error minimization fashion, but fail to achieve the precise optimal control due to an inherent limitation within the controller or control process itself, e.g., delays resulting from processor calculations and sample hold operations in digital systems, or perceptual/neuromuscular lags in the case of a human controller. By letting $H(s)=e^{-s \tau}$, those actual delay limitations displayed by human operators during tracking tasks can be approximated by the parameter $\tau$, an effective transport lag. By incorporating this approximation and noting then that the transfer function relating $u(t)$ and $\epsilon\left(t+T^{*}\right)$ is $e^{-s \tau} /\left(1-e^{-s \tau}\right) K T^{*}$, Fig. 1 reduces to Fig. 2, a single-loop pursuit tracking formulation. The open-loop transfer function $Y_{0}(s)$ relating $y\left(t+T^{*}\right)$ and $\epsilon\left(t+T^{*}\right)$ is given by

$$
Y_{0}(s)=\frac{e^{-s \tau}}{1-e^{-s \tau}}\left[1+\frac{m^{T} \phi\left(t+T^{*}, t\right) G(s)}{K T^{*}}\right] .
$$




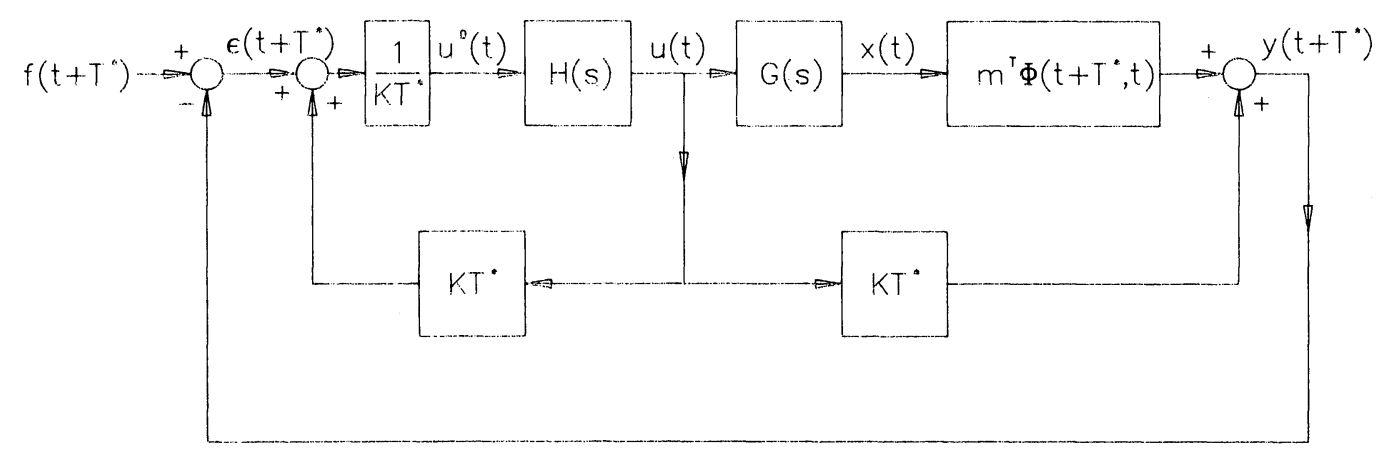

Fig. 1. Block diagram for the single-point preview control.

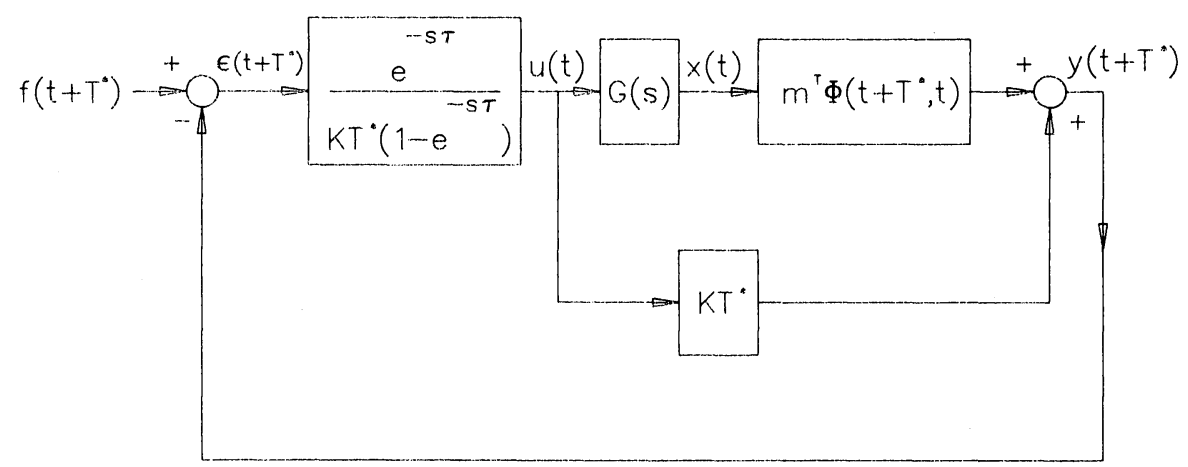

Fig. 2. Equivalent block diagram for the single-point preview control, $H(s)=e^{-s \tau}$.

The stability of this system is determined by the characteristic roots of $1+Y_{0}(s)$, or equivalently,

$$
1+e^{-s \tau} \boldsymbol{m}^{T} \phi\left(t+T^{*}, t\right) \boldsymbol{G}(s) / K T^{*}=0 .
$$

To test the utility of this model by comparison with experimental findings, open-loop gain/phase frequency response results measured by Weir et al. [9, Fig. 12-C] for an automobile straight-line regulatory control task are presented in Figs. 3 and 4. These experimental results represent the open-loop frequency response relating the driver's output (presumably an estimate of future lateral position) to an assumed error, derived by the driver, between the previewed input (straight road ahead) and the driver's output. Since this may be categorized as a form of linear pursuit tracking, the formulation of (11) is accommodated. Also shown in Figs. 3 and 4 is the frequency response calculation for (11) with parameters $T^{*}=3.0(\mathrm{~s})$ and $\tau=$ 0.26 (s). The model output $y\left(t+T^{*}\right)$ is the estimated vehicle lateral position at time $t+T^{*}$; the input $f\left(t+T^{*}\right)$ $\equiv 0$ is the lateral displacement of the previewed path. The automobile $(F, g)$ dynamics used in (11) appear in Appendix I-A and duplicate those identified in [9]. The values of $T^{*}$ and $\tau$ were selected to fit the experimental data as closely as the single-point model would permit. As can be seen, the model and experimental results display excellent agreement. Not only does the preview model reproduce the $--6 \mathrm{db} /$ octave slope of the familiar manual control "crossover" model [2], [8] gain characteristic, but also the peaking phase characteristic usually displayed in manual control task experimental data of this kind.

The model parameters $T^{*}$ and $\tau$ appearing in (11) represent the average preview time used by the driver and

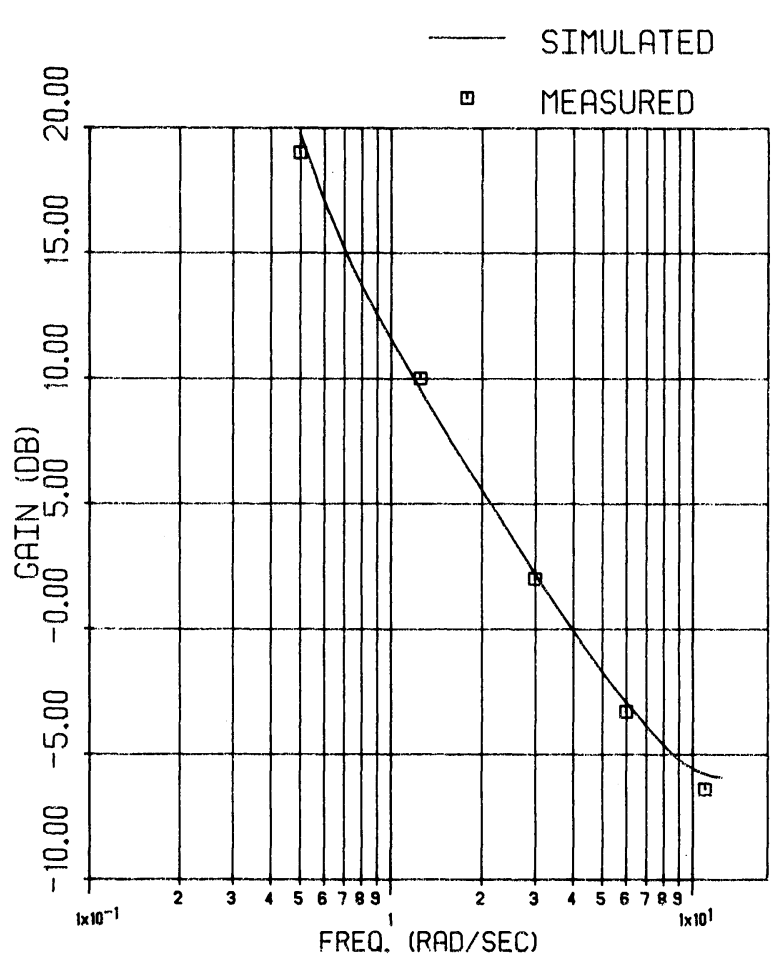

Fig. 3. Frequency response gain comparison.

his/her effective transport lag associated with this particular control task. The values of $T^{*}$ and $\tau$ used here fall well within the range identified by other investigators studying straight-line automobile driving [10]-[12] and human operator tracking performance [2], [4], [9].

Interestingly, for the relatively simple control task of typical straight-line automobile regulation as discussed here, 


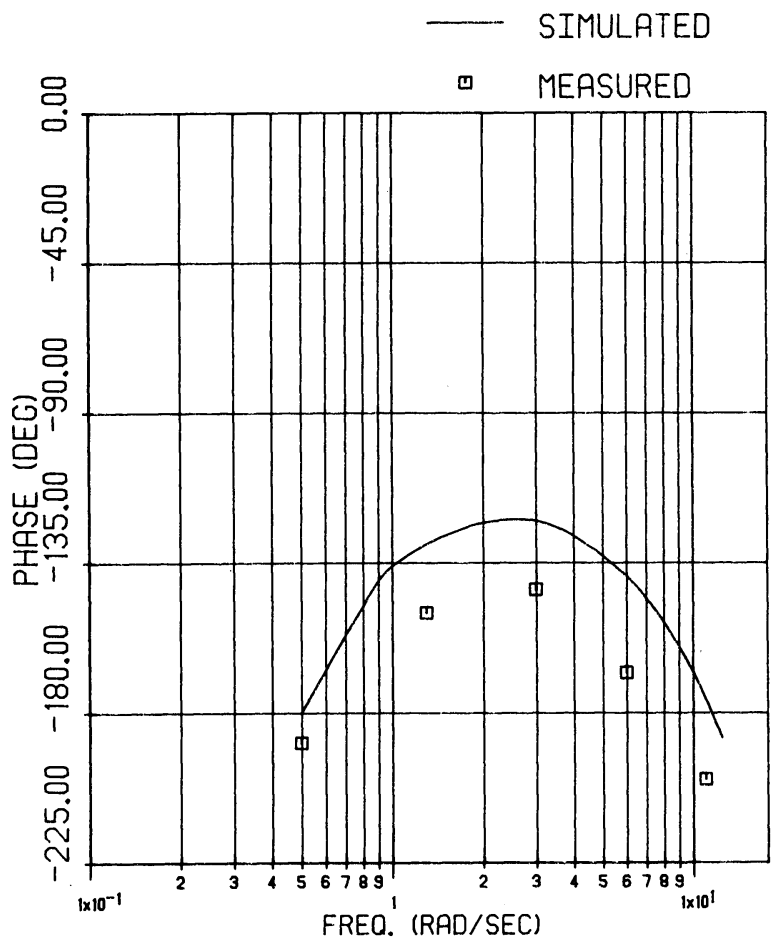

Fig. 4. Frequency response phase comparison.

the vehicle dynamics portion of the total transfer function (11) does not play a dominant role except at very low frequencies. As a result, the open-loop transfer function gain characteristic (11) is closely approximated by the human operator term, $e^{-s \tau} /\left(1-e^{-s \tau}\right) \approx e^{-s \tau} / \tau s$. Such a result would support the well-known fact that tracking task test results for simple automobile regulation [8], [9] can generally be approximated by the "cross-over" model form $C e^{-s \tau} / s$ ( $C$ being the "cross-over" gain constant) in the vicinity of the cross-over frequency. Moreover, in such cases where the above approximation does hold, $1 / \tau$ becomes $C$ in the "cross-over" model representation.

For the simple manual control pursuit tracking task, as represented here by straight-line automobile regulation, the modified optimal preview controller, even employed in only a single-point form $\left[W(\eta-t)=\delta\left(T^{*}\right)\right]$, appears to accurately mimic human control behavior. It might, therefore, seem reasonable to conjecture that human operator strategy during simple pursuit tracking (or at least straightline automobile regulation) is closely akin to an optimal preview error minimization process which ignores or is unaware of transport delay mechanisms inherent in the control processor. A more stringent test of this hypothesis is offered in the following section wherein transient automobile path following is examined using the modified optimal preview control model in its complete form.

\section{Application of the Optimal Preview Control For Simulation of Closed-Loop Transient Automobile Path Following}

The previous section addressed the applicability of the optimal preview control to the problem of preview regulation and the effects of an inherent transport delay within

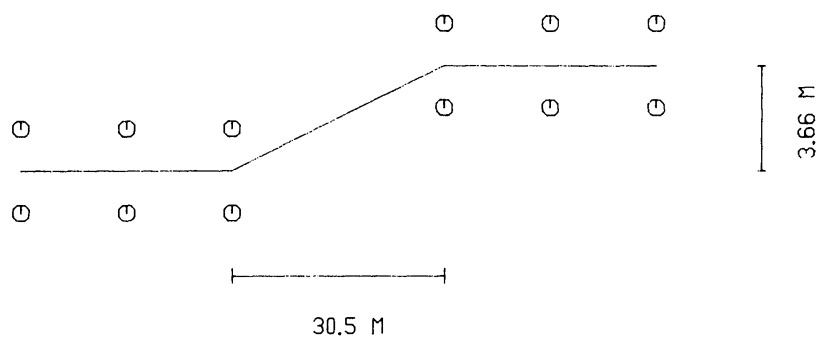

Fig. 5. Lane-change test course.
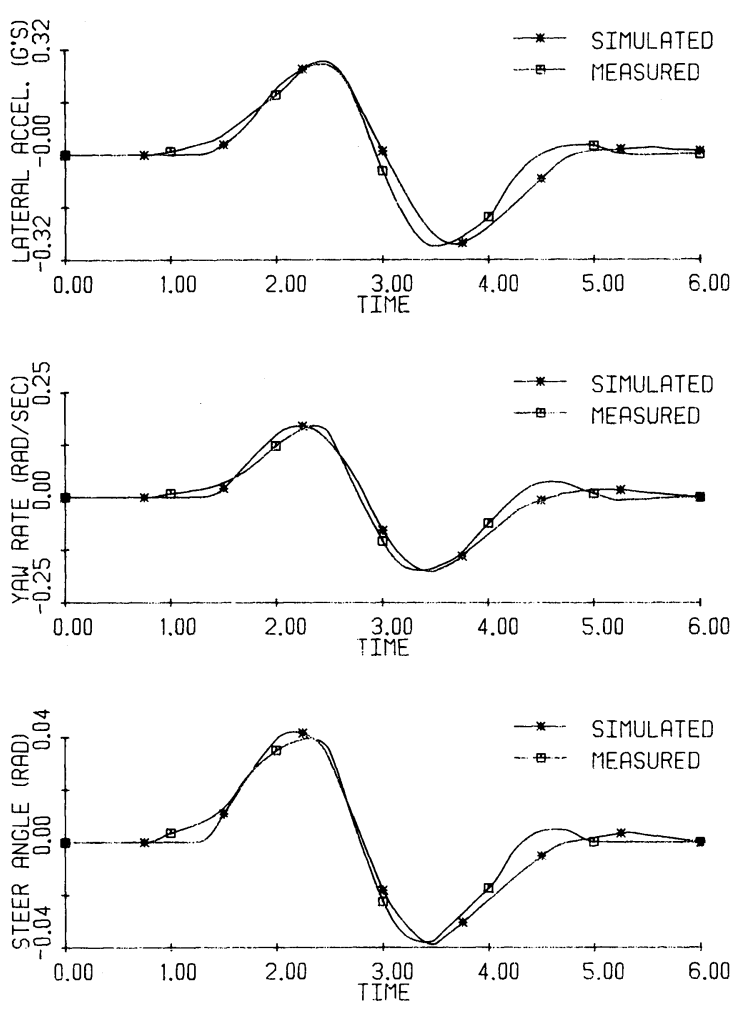

Fig. 6. Closed-loop simulation/test result comparison.

the controller. Using straight-line automobile regulation as an example, the single-point preview model was compared with experimental results within the frequency domain. In this section application to the tracking problem is demonstrated using the general preview control model (7), with an inherent transport time delay to simulate a closed-loop automobile/driver path following maneuver. Results from the model are compared with time history measurements from corresponding full-scale vehicle tests.

The specific closed-loop maneuver examined here required an automobile driver to perform a standard $3.66 \mathrm{~m}$ (12-ft) lane-change within a distance of $30.5 \mathrm{~m}(100 \mathrm{ft})$ at a vehicle speed of approximately $26.8 \mathrm{~m} / \mathrm{s}(60 \mathrm{mi} / \mathrm{h})$. The initiation and completion of the lane change was constrained by $3.05-\mathrm{m}$ wide (10 ft) cone-marked lanes (Fig. 5). The test vehicle was a standard American compact with measured parameter values shown in Appendix I-B. A representative test result for this vehicle/driver combination appears in Fig. 6, showing recorded-time histories of lateral acceleration, yaw rate, and front-wheel steer angle [13]. 
Also shown in Fig. 6 are computer simulation results using the optimal preview control (7) with an assumed human operator transport delay term $e^{-s \tau}$ relating $u^{0}(t)$ and $u(t)$. The transport lag term is included here, as in the previous section, to approximate the principal human operator lag effects. The calculation of (7), steer angle, seen in Fig. 6 is for values of $\tau=0.2$ (s) and $T=1.3$ (s) using ten equally spaced points in the preview interval to approximate the integral. The values of $T$ and $\tau$ were selected to closely fit the test measurements. The $(F, \boldsymbol{g})$ automobile dynamics model is the same two-degree-of-freedom model appearing in Appendix I-A, evaluated for the parameter values identified in Appendix I-B. The previewed input $f(\eta)$ appearing in (7) represents the desired lateral path deviation and was obtained during the simulation using the simple straight-line path segments shown in Fig. 5 as input.

As seen from Fig. 6, excellent agreement can be obtained between the experimental results and simulation predictions using the two numerical parameters $(\tau, T)$ and a simple straight-line path input. Variations in the value of $\tau$ primarily influenced the closed-loop system damping; larger values producing reduced damping. Variations in the value of $T$ influenced control (steering) amplitude as well as damping; larger values of $T$ producing lower control amplitude and increased damping.

Finally, Fig. 7 shows a comparison of the preview model predictions and measured test results for a modified set of vehicle dynamics $(F, g)$. The same vehicle was employed but with modifications to its mass center and rear tires so as to produce a new set of parameter values listed in Appendix I-C. As shown in Fig. 7 the principal change in the closed-loop response from Fig. 6 is an increased steering gain (lower steering amplitude for the same nominal maneuver) and decreased damping. Larger values of $\tau(0.3)$ and $T(1.55)$ were required in the calculation of (7), shown as steer angle in Fig. 7, to better approximate the reduced damping and smaller amplitude steering control. A comparison of computed vehicle path trajectories, corresponding to the baseline and modified vehicle responses shown in Figs. 6 and 7, appears in Fig. 8.

Characteristic roots for each of the closed-loop systems, as calculated from the constant matrix (13), are shown in Fig. 9. The matrix (13) (see Appendix I-D) is similar to that given by (9) but includes the influence of the transport lag term $e^{-s \tau}$ approximated by the first-order Padé polynomial

$$
\begin{gathered}
\frac{1-\frac{\tau}{2} s}{1+\frac{\tau}{2} s} \\
{\left[\begin{array}{c}
F \\
c^{T}\left(F-\frac{2}{\tau} I\right) \\
\hdashline c^{T} g-\frac{g}{\tau}
\end{array}\right] .}
\end{gathered}
$$

Note that the reduced damping in the driver/vehicle responses, displayed in Figs. 7 and 8, is equivalently represented by the corresponding closed-loop characteristic root locations shown in Fig. 9.
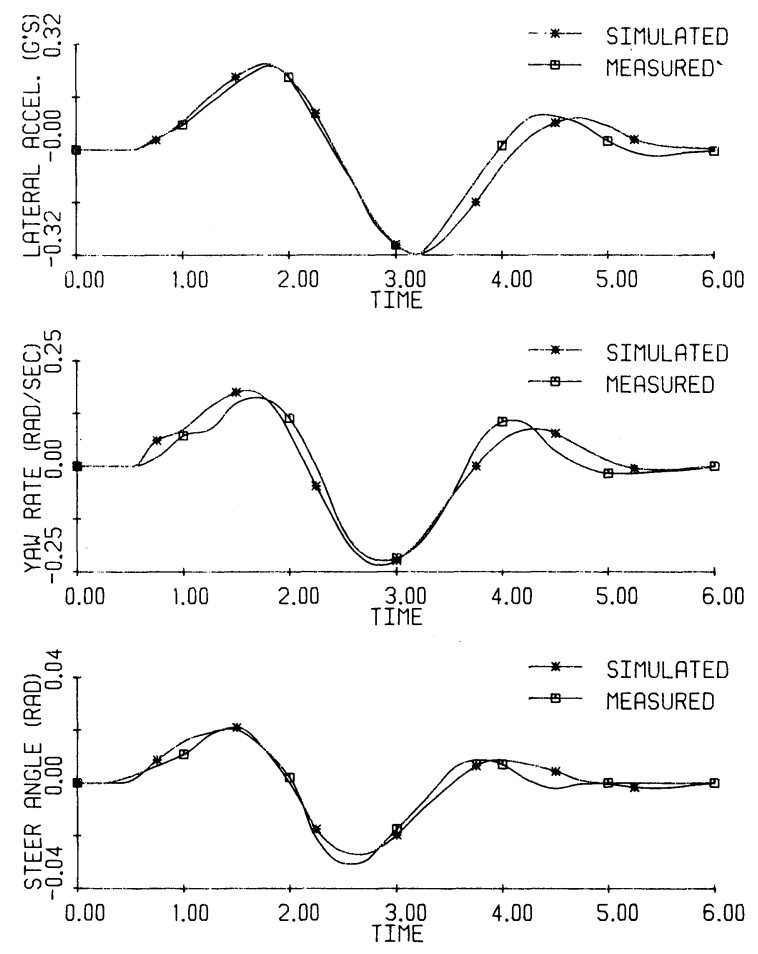

Fig. 7. Closed-loop simulation/test result comparison-modified vehicle.

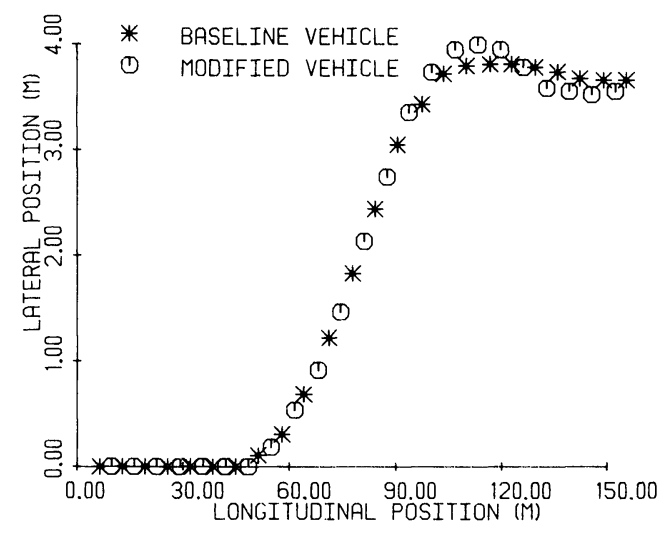

Fig. 8. Simulated path trajectories.

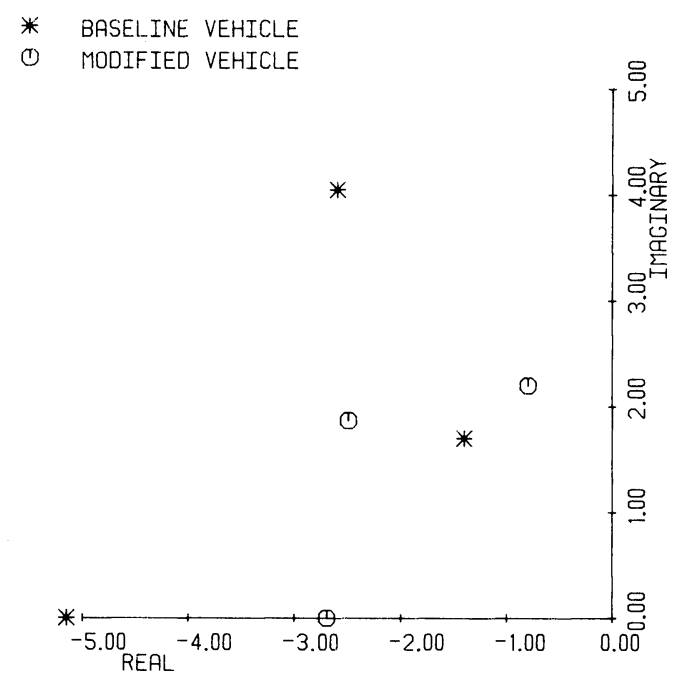

Fig. 9. Characteristic roots of the baseline and modified closed-loop systems. 
These results and those of the previous section demonstrate useful application of the optimal preview model in simulation of closed-loop automobile driving. The principal conclusion concerning these results is that driver steering control strategy during path following can be accurately represented as a time-lagged optimal preview control. Similar applications and extensions to problems in other fields are clearly suggested by the results shown here.

\section{CONCLUSION}

The optimal preview control model, applied here to the closed-loop automobile path following problem, offers a useful and direct method for representing closed-loop behavior of linear driver/vehicle systems. It is suggested that driver automobile steering control strategy during path following can be viewed as a time-lagged optimal preview control process.

The general linear system formulation of the preview control methodology, demonstrated here, permits application to a broad range of problems relating to manmachine systems.

\section{APPENDIX I}

\section{A. Vehicle Dynamics}

The linear dynamical equations of an automobile for lateral and yaw motions are

$$
\begin{aligned}
& \dot{y}=v+U \psi \\
& \dot{v}=\left[-2\left(C_{\alpha_{F}}+C_{\alpha_{R}}\right) / m U\right] v+\left[2\left(b C_{\alpha_{R}}-a C_{\alpha_{F}}\right) / m U-U\right] r \\
& +\left(2 C_{\alpha_{F}} / m\right) \delta_{F W} \\
& \dot{r}=\left[2\left(b C_{\alpha_{R}}-a C_{\alpha_{F}}\right) / I U\right] v+\left[-2\left(a^{2} C_{\alpha_{F}}+b^{2} C_{\alpha_{R}}\right) / I U\right] r \\
& +\left(2 a C_{\alpha_{F}} / I\right) \delta_{F W} \\
& \dot{\psi}=r
\end{aligned}
$$

where

$y \quad$ inertial lateral displacement of the vehicle mass center,

$v$ lateral velocity in the vehicle body axis system,

$r \quad$ yaw rate about the vertical body axis,

$\psi \quad$ vehicle heading angle, and

$\delta_{F W}$ front tire steer angle, control variable.

The parameters appearing in (A1)-(A4) are

$U$ forward vehicle velocity,

$C_{\alpha_{F}}, C_{\alpha_{R}}$ front and rear tire cornering coefficients,

$a, b \quad$ forward and rearward locations of tires from the vehicle mass center, and

$m, I \quad$ vehicle mass and rotational inertia.

The above equations can be expressed in matrix notation as

$$
\dot{\boldsymbol{x}}=F \boldsymbol{x}+\boldsymbol{g} \delta_{F W}
$$

where

$$
\begin{array}{ll}
\boldsymbol{x} & =\left\{\begin{array}{l}
y \\
v \\
r \\
\psi
\end{array}\right\} \\
F & =\left[\begin{array}{llll}
0 & 1 & 0 & U \\
0 & A_{1} & B_{1} & 0 \\
0 & A_{2} & B_{2} & 0 \\
0 & 0 & 1 & 0
\end{array}\right], \quad \boldsymbol{g}=\left\{\begin{array}{l}
0 \\
C_{1} \\
C_{2} \\
0
\end{array}\right\}
\end{array}
$$

and

$$
\begin{aligned}
& A_{1}=-2\left(C_{\alpha_{F}}+C_{\alpha_{R}}\right) / m U \\
& B_{1}=2\left(b C_{\alpha_{R}}-a C_{\alpha_{F}}\right) / m U-U \\
& C_{1}=2 C_{\alpha_{F}} / m \\
& A_{2}=2\left(b C_{\alpha_{R}}-a C_{\alpha_{F}}\right) / I U \\
& B_{2}=-2\left(a^{2} C_{\alpha_{F}}+b^{2} C_{\alpha_{R}}\right) / I U \\
& C_{2}=2 a C_{\alpha_{F}} / I .
\end{aligned}
$$

The calculation of (11) appearing in Figs. 3 and 4 used the following parameter values identified in [9] for vehicle D

$$
\begin{aligned}
a & =1.41 \mathrm{~m}(4.63 \mathrm{ft}) \\
b & =1.41 \mathrm{~m}(4.63 \mathrm{ft}) \\
m & =2016 \mathrm{~kg}(138 \mathrm{slug}) \\
I & =4013 \mathrm{~m} \cdot \mathrm{N} \cdot \mathrm{s}^{2}\left(2960 \mathrm{ft} \cdot \mathrm{lb} \cdot \mathrm{s}^{2}\right) \\
U & =22.3 \mathrm{~m} / \mathrm{s}(73.3 \mathrm{ft} / \mathrm{s}) \\
C_{\alpha_{F}} & =25266 \mathrm{~N} / \mathrm{rad}(5680 \mathrm{lb} / \mathrm{rad}) \\
C_{\alpha_{k}} & =70933 \mathrm{~N} / \mathrm{rad}(15960 \mathrm{lb} / \mathrm{rad}) .
\end{aligned}
$$

The constant observer vector $\boldsymbol{m}^{r}=(1,0,0,0)$ provided the vehicle lateral position $y$.

\section{B. Baseline Vehicle Parameter Values}

The vehicle parameter values listed below and used in the calculations appearing in Fig. 6 were derived from vehicle wheelbase/weight measurements and steady-state, constant-steer vehicle test results [13]

$$
\begin{aligned}
a & =1.37 \mathrm{~m}(4.5 \mathrm{ft}) \\
b & =1.22 \mathrm{~m}(4.0 \mathrm{ft}) \\
m & =1563 \mathrm{~kg}(107 \mathrm{slug}) \\
I & =2712 \mathrm{~m} \cdot \mathrm{N} \cdot \mathrm{s}^{2}\left(2000 \mathrm{ft} \cdot \mathrm{lb} \cdot \mathrm{s}^{2}\right) \\
U & =25.9 \mathrm{~m} / \mathrm{s}(85 \mathrm{ft} / \mathrm{s}) \\
C_{\alpha_{F}} & =19438 \mathrm{~N} / \mathrm{rad}(4370 \mathrm{lb} / \mathrm{rad}) \\
C_{\alpha_{R}} & =33628 \mathrm{~N} / \mathrm{rad}(7560 \mathrm{lb} / \mathrm{rad}) .
\end{aligned}
$$

The weighting function $W$ appearing in (7) was selected as constant 1.0 over the ten-point preview interval.

\section{Modified Vehicle Parameter Values}

The vehicle parameters of Appendix I-B were altered to those values shown in this section by a rearward shift in the vehicle mass center and a decrease in rear tire inflation 
pressures

$$
\begin{aligned}
& a=1.43 \mathrm{~m}(4.7 \mathrm{ft}) \\
& b=1.16 \mathrm{~m}(3.8 \mathrm{ft}) \\
& m=1753 \mathrm{~kg}(120 \mathrm{slug}) \\
& I=2712 \mathrm{~m} \cdot \mathrm{N} \cdot \mathrm{s}^{2}\left(2000 \mathrm{ft} \cdot \mathrm{lb} \cdot \mathrm{s}^{2}\right) \\
& U=25.9 \mathrm{~m} / \mathrm{s}(85 \mathrm{ft} / \mathrm{s}) \\
& C_{\alpha_{F}}=20906 \mathrm{~N} / \mathrm{rad}(4700 \mathrm{lb} / \mathrm{rad}) \\
& C_{\alpha_{R}}=29536 \mathrm{~N} / \mathrm{rad}(6640 \mathrm{lb} / \mathrm{rad}) .
\end{aligned}
$$

The closed-loop calculation using these parameter values appears in Fig. 7.

\section{Stability of the Closed-Loop Optimal Preview-Controlled} System Including a Transport Time Lag

Given the system

$$
\begin{aligned}
& \dot{x}=F x+g u \\
& u=e^{-s \tau} u^{0} \\
& u^{0}=-c^{T} x
\end{aligned}
$$

where $F, g, u^{0}$, and $c^{r}$ are defined in (1), (4), and (9). If the transport time lag $e^{-s \tau}$ is approximated by the first-order Padé polynomial,

$$
\frac{1-\frac{\tau}{2} s}{1+\frac{\tau}{2} s}
$$

(A7) becomes

$$
\dot{u}=\frac{2}{\tau}\left(-u+u^{0}\right)-\dot{u}^{0}
$$

Substitution of

$$
u^{0}=-c^{T}
$$

and

$$
\dot{u}^{0}=-c^{T}[F x+g u]
$$

into (A10) produces the closed-loop state equation

$$
\left\{\begin{array}{c}
\dot{\boldsymbol{x}} \\
\hdashline \dot{u}
\end{array}\right\}=\left[\begin{array}{c:c}
F & g \\
\hdashline c^{T}\left(F-\frac{2}{\tau} I\right) & c^{T}-\frac{2}{\tau}
\end{array}\right]\left\{\begin{array}{c}
x \\
\hdashline u
\end{array}\right\}
$$

equivalent of (A6)-(A8). For small $\tau$, stability of the time-lagged optimal preview-controlled system is provided by the characteristic roots of the system matrix appearing in (A11).

\section{REFERENCES}

[1] C. C. MacAdam, "An optimal preview control for linear systens," J. Dynamic Systems, Measurement, Control, Sept., 1980.

[2] D. T. McRuer, et al., "New approaches to human-pilot/vehicle analysis," Systems Technology, Inc., Tech. Rep., AFFDL-TR-67150, Feb. 1968

[3] W. W. Wierwille, G. A. Gagne, and J. R. Knight, "An experimental study of human operator models and closed-loop analysis methods for high-speed autombile driving," IEEE Trans. Hum. Factors Electron., vol. HFE-8, no. 3, pp. 187-201, Sept. 1967.

[4] K. Tanaka, N. Goto, and K. Washizu "A comparison of techniques for identifying human operator dynamics utilizing time series analysis," in Proc. Twelfth Annu. Corif. Manual Control, Univ. of Illinois, Urbana, IL, May 25-27, 1976, pp. 673-693.

[5] D. H. Weir, "Motorcycle handling dynamics and rider control and the effect of design configuration on response and performance," Ph.D. dissertation, Univer. of California, Los Angeles, CA 1972.

[6] S. Ben-Ari and J. R. Ellis, "The control of an articulated semitrailer vehicle," in Vehicle Safety Legislation-Its Engineering and Social Implications. London: Mechanical Engineering Publications Limited, 1975.

[7] D. L. Kleinman, S. Baron, and W. H. Levison, "An optimal control model of human response, part I: Theory and validation," Automatica, vol. 6 , pp. $357-369,1970$.

[8] D. T. MicRuer et al., "New results in driver steering control models," Human Factors, vol. 19, pp. 381-397, Aug. 1977.

[9] D. H. Weir, R. J. DiMarco, and D. T. McRuer, "Evaluation and correlation of driver/vehicle data," vol. II, Final Tech. Rep., National Highway Traffic Safeiy Admin., DOT-HS-803-246, Apr. 1977.

[10] R. G. Mortimer and C. M. Jorgeson, "Eye fixations of drivers as affected by highway and traffic characteristics and moderate doses of alcohol," in Proc. Sixteenth Annu. Meeting, Human Factors Society, Oct. 17-19, 1972, pp. 86-92.

[11] M. Kondo and A. Ajimine, "Driver's sight point and dynamics of the driver-vehicle-system related to it," SAE Paper No. 680104, Automotive Engineering Congress, Detroit, MI, Jan. 8- 12, 1968.

[12] D. A. Gordon, "Experimental isolation of drivers' visual input," Public Roads, vol. 33, pp. 266-273, 1966.

[13] "Comparison of vehicle test procedures," Contract DRDA 781433, Highway Safety Research Institute, University of Michigan, 1978 